\title{
Recrystallization of Drugs: Significance on Pharmaceutical Processing
}

\author{
Yousef Javadzadeh, Sanaz Hamedeyazdan and Solmaz Asnaashari \\ Biotechnology Research Center and Faculty of Pharmacy, \\ Tabriz University of Medical Sciences
}

Iran

\section{Introduction}

Not surprisingly, the wide range of effective medicinal agents available today is one of the greatest scientific achievements. Regardless of the advancements in effectiveness and safety of the medicines embedded in dosage forms, the pharmaceutical concept of the latter is growing to be ever more eminent (Adibkia et al., 2011). Following on from recent advancements, in a time of increased considerations to the level of sophistication in designing pharmaceutical dosage forms keeping pace with advances in drug discovery methods, it seems as important as ever to study the physicochemical properties of active pharmaceutical ingredients, prerequisite for a successful product formulation.

As far as we know, the molecular structure of any drug compound typically defines all of its physical, chemical and biological actions. Owing to the fact that a certain kind of drug might be offered in a variety of solid forms, including polymorphs, solvates, hydrates, salts, cocrystals and amorphous solids, the choice and design of the ideal solid-state chemistry of the pharmaceutical solid form would be critically important to a superior drug development. Accordingly, drug crystals could be modified in different ways including recrystallization, which would affect the physical and physicochemical properties such as melting point, solubility, true density, drug release profile, flowability and tabletability of the pharmaceutical dosage forms (Harbury, 1947; JamaliMitchell, 1973; Jozwiakowski et al., 1996).

Recrystallization is a simple and inexpensive method for scaling up the drug developments to a commercial level. Significant advances in the different pharmaceutical dosage form technologies renders drug recrystallization as a green technique due to the savings of costs, time, energy and less machinery as well as fewer personnel. Recrystallization is one aspect of precipitation obtained through a variation of the solubility conditions and the amount of dissolved solute in an increased temperature. In general, production of another crystalline from of a drug and also purification are the two major sets of applications for drug recrystallization processes. Briefly, in a drug recrystallization process, a hot saturated solution of the drug is prepared with only enough solvent to dissolve it at the boiling point of the solution. Once the solution is cooled the purified drug component or a new crystal form of the drug separates as a result of the lower solubility of that crystalline form of a drug in the respective solvent at lower temperatures. 
Since impurities are present in fairly small amounts of drug solutions they do not crystallize in recrystallization and they are ready to separate from the formed drug crystal (Tiwary, 2001). For instance, in the case of natural medicinal compounds obtained from natural sources which almost always contain impurities, in order to obtain a pure drug, usual major steps in the recrystallization process have been schematically demonstrated in figure 1 . Purifying a sample drug of compound $X$ which is contaminated by a small amount of compound $Y$, would be established with an appropriate hot solvent in which all of compound $Y$ is soluble at room temperature and the impurities will stay insoluble in and pass through filter paper, leaving only pure drug crystals behind, as has been shown in figure 1 .

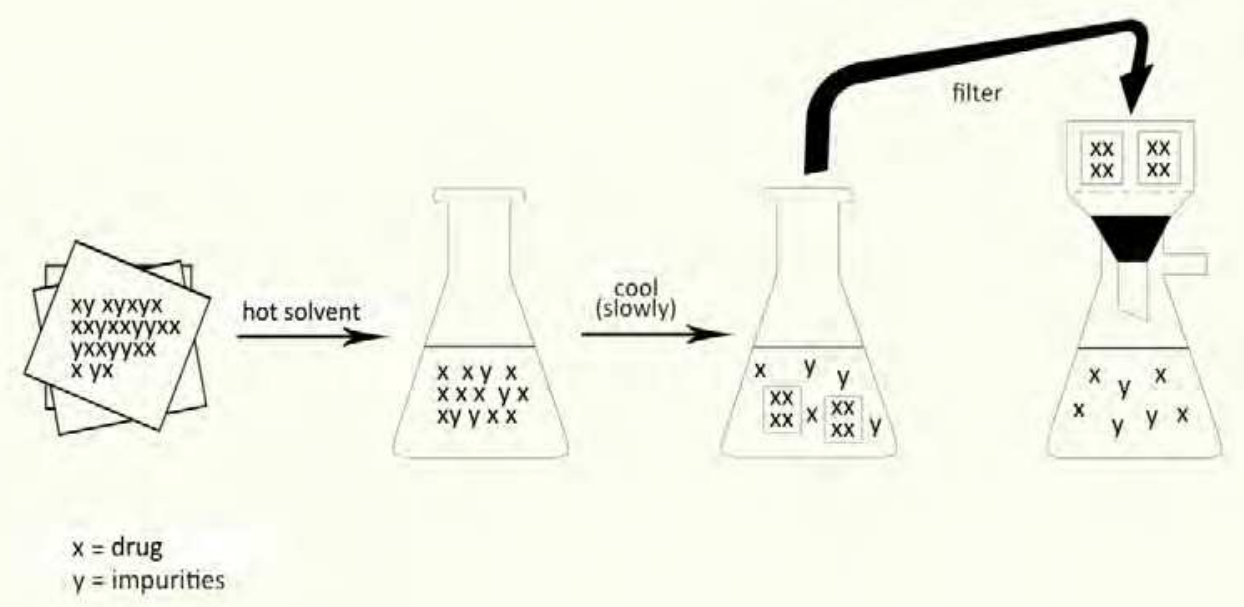

Fig. 1. Schematic protocol of drug recrystallization for further purification.

It is worth mentioning that in any recrystallization technique some drug loss is inevitable and the total recovery would be less than $100 \%$, seeing that even at the lower temperatures the target drug has some finite solubility in the solvent and is lost subsequently when solvent and soluble impurities are removed. Moreover, selection of the right solvent in the recrystallization seems to be one of the crucial features of the process and is made on a caseby-case basis (MirmehrabiRohani, 2005; ChenTrout, 2008). This is because of solubility variation of different drug compounds in different solvents so that a certain drug not only should have the highest solubility in the solvent of choice at its boiling point, but also it should show a markedly diminished solubility at lower temperatures of the same solvent. Although recrystallization is a very common technique used to purify drugs, it has a basic limitation for the compounds that are mostly pure and other techniques of separations are of use for the drug mixtures containing several major components which could not be purified by recrystallization methods.

Ever since, the leading physicochemical properties of a unique form of a drug could seriously influence the bioavailability, manufacturability purification, stability, solubility, and other characteristics, identifying these potential liabilities allows us to predict, control 
and avoid any complexities that may arise during drug development stages (Krishnaiah, 2010). This would be beneficial in preventing the drug development efforts to costly late stage product failures throughout the manufacture and storage periods too. However, in order to fully control the crystallization process, the link between a particular solid form of a drug structure and its functional physicochemical properties, still challenges to be better established to facilitate the suitable drug production. A large and growing body of literatures has been published on recrystallization techniques and physicochemical properties of a drug. Herein, we gathered some of the related reports of drug recrystallization to have an overlook on the crystal habit of a drug on some basic physical properties of pharmaceutical dosage forms signifying how these factors are interrelated.

\section{Impact of crystal habit on pharmaceutical processing}

Drug discovery and characterization relies on the nature of the target molecule and the relative physicochemical properties of drugs. Identifying all relevant crystal habit of a drug which is an important variable in pharmaceutical manufacturing at the development phase from research to commercialization is of substantial value. Due to the different crystal form variations of some basic physical properties like, solubility, dissolution rate, melting behavior, and certain micromeritic properties or performance characteristics, e.g. tablet compressibility, mechanical strength, powder flow provide alternatives to select a form that presents the suitable balance of critical properties for development into the drug product. Establishing such modification information at an early stage of drug development process lessens the risk of process alterations given form changes and brings in the opportunity to attain more comprehensive rational property coverage.

The merit of changes in crystal surface form and habit of drug powders by recrystallization method is much more realized when there is an essential to diminish variations in raw material characteristics, to certify reproducibility of results during drug preformulation, and also to judge fairly about the cause of poor performance of a dosage form. Besides, the changes in crystal habit of a drug going together with or without polymorphic transformation at some point in processing storage could account for serious implications of physical stability in dosage forms. Thus, it seems underlying to have a deeper insight to the crystal structures and control the solid-state chemistry of drug substances to design a more systematic and intellectual pharmaceutical dosage forms.

In a survey carried out by Sinclair et al. ibipinabant a potent and highly selective cannabinoid receptor antagonist was evaluated for its solid-state physical stability and recrystallization kinetics in tablet dosage forms using fourier transform raman spectroscopy. The findings of the study showed that exposure to moisture had notable influence on the crystallinity of amorphous ibipinabant. The recrystallization kinetics measurements revealed a two-step process with an induction period (nucleation) followed by rod-like crystal growth by application of the Johnson-Mehl-Avrami kinetic model. On the whole their method provided reliable and highly accurate predictive crystallinity assessments after exposure to a variety of stability storage conditions for ibipinabant (Sinclair et al., 2011).

Recently, Dahlberg et al. analyzed the stability of the amorphous drug, flutamide, by a combination of localized nuclear magnetic resonance (NMR) spectroscopic and NMR imaging techniques. Owing to the fact that, NMR relaxation is sensitive to both the 
crystalline and amorphous state and the size of the drug substance, it allows for an in situ monitoring of the state of the drug during tablet disintegration and dissolution periods. With regard to the results of the NMR experiments, recrystallization was believed to be related to its enabling factors such as local hydration level and local mobility of the polymer matrix. Eventually, it was verified that the primarily amorphous flutamide may recrystallize either by nanoparticle coalescence or by ripening of crystalline particles (Dahlberg et al., 2011).

The solid-state properties of sulfathiazole and chlorpropamide were modified through recrystallization using supercritical antisolvent process by Yeo et al. They confirmed that the operating conditions of the system such as carbon dioxide injection rate, type of solvent, and temperature significantly had an effect on the physical characteristics of the resulting crystals. Considering the results of the study, drug crystals processed with supercritical system exhibited more ordered appearances with clean surfaces and sharp angles compared with the unprocessed particles where crystal habit changed from tabular to acicular when the carbon dioxide injection rate increased. Photomicrographs of sulfathiazole crystals with methanol as a solvent, confirmed a needle-like acicular and a tabular crystal habits in rapid and slow injections, respectively. Whereas, in the case of chlorpropamide, processed drug particles in the rapid injection experiment exhibited columnar habit in a regular shape, while relatively large crystals with sharp angles were observed in the slow injection mode when acetone was used as the solvent. Overall experimental observations suggested that the supercritical antisolvent process could provide favorable environment for the solid growth of a single type of crystalline drug, minimizing the conditions for growth-related imperfections (Yeo et al., 2003).

According to the fact that thermal analysis has been frequently used to identify crystal forms of drugs and in the course of thermal analysis, crystal transformation is often observed as well as melting and decomposition, Suzuki et al. studied mechanisms of thermal crystal transformation through melting and recrystallization. They characterized two anhydrates ( $\alpha$-from and $\beta$-from) and two hydrates (hemihydrate and monohydrate) forms of a novel fluoroquinolone antibiotic, sitafloxacin, in addition to sesquihydrate which is used in the marketed drug products. The results of crystal structural that were characterized by infrared spectroscopy, X-ray powder diffractometry and thermal analysis revealed quinolone rings of sitafloxacin had distorted planar structure and quinolone ring of the drug in a-form and monohydrate hold opposite torsion to those in $\beta$-form and sesquihydrate. These kinds of thermal analysis are often recommended as a routine tool for quality control of thermal dehydration and subsequent crystal conversion of drugs (Suzuki et al., 2010).

\subsection{Compaction and flowability}

Acquiring a clear notion of why certain drug materials are prone to problems during compaction and dominate the relative constraints to offer a successful compaction and tableting strategy of pharmaceutical powders would involve an understanding of the fundamental properties of drug powders. Therefore, it is important to determine the effect of different physicochemical properties such as particle size, shape, surface area, polymorphic form, crystal habit, hydrates, and processing conditions on the compaction of powders. As different crystal habits of a certain drug hold dissimilar planes, they have differing points in their specific surface and free surface energies. Even so, alternative 
recrystallization solvents could develop crystal with defined crystal habit, size and shape as well as compressibility properties. The nature and amount of these changes count on the recrystallization conditions including the presence of impurities, type of solvents and cooling rates. This view is supported by a variety of papers at molecular level developing the knowledge of solid-state properties such as crystal structure, crystal habit, and polymorphism influence on the mechanical properties of powders in an attempt to identify and modify physical properties of bulk solids of drugs (Liebenberg et al., 1999; Maghsoodi et al., 2007).

Seton et al. evaluated the particle morphology of ibuprofen, an anti-inflammatory drug, by recrystallization from a range of solvents and investigated the following influence on compaction properties. The compaction data achieved from properties of the ibuprofen control and recrystallized samples at different compaction forces and speeds revealed equal or better tablet strength than the control, whilst ibuprofen recrystallized from 2-ethoxyethyl acetate exhibited lower levels of elastic energy during compaction. In addition, the recrystallized ibuprofen samples demonstrated flowablity equivalent quality to the ibuprofen control, excluding the ibuprofen recrystallized from acetone which showed excellent flow properties. Generally, the results displayed ibuprofen recrystallization from various solvents could offer advantages in terms of particle morphology, flowability and compaction properties (Seton et al., 2010).

In another study an anti-epileptic drug, phenytoin, crystals in the form of free acid, having distinct types of habits, was modified via different recrystallization conditions and techniques by Nokhodchi et al. Several sets of experimental conditions for temperature, solvent evaporation and watering-out techniques were applied for evaluation of the drug recrystallization in ethanol and acetone solvents. The solid state characteristics and compaction properties of the crystal habit with factors affecting the resultant crystals were also evaluated. The physical characteristics of the crystals were investigated using scanning electron microscopy, X-ray powder diffractometry, FT-IR spectrometry and differential scanning calorimetry. They confirmed that using watering-out technique as a crystallization method, produced thin plate crystals, while the crystals obtained by other methods were needle shape for alcoholic solutions and rhombic for acetone solutions. Although the crystallization medium had central effect on phenytoin crystal habit modification, altering crystallization temperature had no effect on crystal habits except a change in size of crystals. In the case of compaction, the crystals produced from alcohol or acetone showed high crushing strengths as a result of lower porosity and lower elastic recoveries (Nokhodchi et al., 2003).

As we know, ascorbic acid crystals are unsuitable for direct tableting due to their poorly compactible properties, Kawashima et al. designed spherically agglomerated crystals of ascorbic acid with improved compactibility for direct tableting. They precipitated ascorbic acid crystals by a solvent change method, followed by their agglomerations with the emulsion solvent diffusion or spherical agglomeration mechanism, depending on the solvent combination for crystallization. Considering the results of the study, under static compression, effectively the proper compact with a sufficient strength was produced. After all improved micromeritic properties, such as flowability and packability for the spherically agglomerated crystals were obtained for crystals of ascorbic acid with the spherical crystallization technique (Kawashima, 2003). 
Designing a suitable dosage form with an ideal physicochemical and mechanical property is an important basic principle of drug delivery systems. As follows, crystal structure, shape, and size of drug substances have a huge economical and practical effect at all stages of development from research to commercialization. So, there is a necessity to control the critical properties of drugs for their readiness and capacity to form a tablet which are dominating dosage forms in pharmaceutical dosage form manufacturing.

\subsection{Solubility, dissolution, and bioavailability of drugs}

Nowadays, in pharmaceutical companies drugs with restricted aqueous solubility have become ever more prevalent and challengeable in the research and development stages. Slow drug dissolution in biological fluids, insufficient and inconsistent systemic exposure and subsequent inadequate efficacy in patients, are some of routine challenges to be coped with during the development of poorly water-soluble drug substances especially when they are administrated orally. Notable numbers of drugs especially new drug candidates are in a biopharmaceutical classification of low solubility (BCS Class II and IV) keeping drug dissolution rate as the limiting factor for the drug absorption and attaining suitable bloodlevels of the drugs (LobenbergAmidon, 2000). These inadequacies in solubility of clinically established drug substances in water and in the gastric fluids make problems in drug dissolution rate and oral bioavailability of drugs, as well (Blagden et al., 2007). Consequently, there is a basic requisite to deliver such drugs in a way that gives a chance of sufficient dissolution rate, absorption, and demonstrating suitable clinical efficacy.

Numerous scientific and technological advancements have been made in the research and development for improving and maximizing dissolution rates of the mentioned types of drugs. Despite enhancements in solubility and dissolution rate and oral bioavailability of poorly water-soluble drugs with the customary pharmaceutical technologists, still there are concerns about the success of those methods in the complexities arise from the specific physicochemical nature of the drug molecule itself (Krishnaiah S.R., 2010). One of the thriving trends in enhancing the solubility, dissolution rate and subsequent bioavailability of poorly soluble drugs is to deal with crystal forms of materials which could potentially be applicable to a broad range of drugs with different crystalline habits (Yeo, Kim et al., 2003). On account of many factors such as crystal habit, size and even polymorphic forms of a drug, dissolution rates would enhance through habit recrystallization. A number of reports in the literature validated the effects of crystal morphology variation on solubility, in vitro dissolution rate, holding potentials for improving drug bioavailability (Kawashima et al., 1986; Carino et al., 2006).

Several studies in this filed have shown that exposure of diverse crystal faces determines the nature of the wettability and consequent enhancements in dissolution rate of the drugs with different crystalline shapes (Heng et al., 2006). In 2000, Kobayashi et al. published a paper in which they presented different dissolution rates for carbamazepine, where the dihydrate form of the drug in simulated fluids ( $\mathrm{pH}$ 1.2) had notably slower dissolution rates than the anhydrous forms (forms I and III). Although the metastable polymorph (Form III) possessed greatest rates of dissolution at the initial stages, reductions in dissolution rate at later time points of the profile was achieved due to the rapid conversion of metastable polymorph (Form III) to the dihydrate. Nevertheless, in another study carried out by Tian et al. the behavior of carbamazepine and dihydrate 
compacts during in vitro dissolution tests various factors were evaluated. Considering the results, presence of excipients such as polyethylene glycol (PEG) and hydroxyl propyl methyl cellulose (HPMC) inhibited the conversion of carbamazepine to the hydrated form following decreased rates of drug dissolution (Tian et al., 2007). Application of different drug habits in pharmaceutical dosage forms could vary the dissolution rates, as the use of metastable polymorphs in enhancing drug dissolution rates. They also performed bioavailability tests in dogs to determine the effects of physicochemical properties of drug form I, form III and dihydrate on the plasma level of carbamazepine. Similar to the findings of other dissolution studies for carbamazepine, drug bioavailability that had been measured was lowest for the dehydrate form. The lower drug bioavailability established with metastable form was in consistent with the probable conversion of the drug habit to the dihydrate (Kobayashi et al., 2000).

Intrinsic solubility of three crystals of diclofenac, a nonsteroidal anti-inflammatory drug, was investigated by Llinas et al. The crystal habits were characterized and detected by thermo gravimetric analysis, differential scanning calorimetry, and X-ray diffraction. They recrystallized the anhydrous sodium salt of commercially available diclofenac with ethanol and precipitated as a hydrated drug that provided consistent results for the intrinsic solubility. Regarding the broad range of values which have been reported for aqueous diclofenac solubility in the literature, they claimed their solubility records were at the smaller end of the range (Llinas et al., 2007).

Perlovich et al. analyzed four new crystal structures of the sulfonamides by X-ray diffraction experiments and comparative analysis of molecular conformational states and hydrogen bonds networks by graph set notations in the crystal lattices. They established temperature dependencies of the solubility in water, $n$-octanol as well as thermodynamic functions of solubility and solvation processes for the compounds. According to Perlovich et al. distinguishing between enthalpy and entropy leads to the insight that the mechanism is different for the different molecules where it may be of importance for further assessment of distribution of drug molecules and provide a better understanding of biopharmaceutical properties of drugs (Perlovich et al., 2008).

Dipyridamole as a critical antiplatelet and peripheral vasodilator drug is known to have properties of water insolubility and poor bioavailability which are the limitations of its effectiveness in clinical usage. Adhiyaman et al. characterized dipyridamole crystals with different types of habits by recrystallization from selected solvents. Physicochemical characteristics of the crystals were assessed via scanning electron microscopy, X-ray powder diffractometry, IR spectrometry and differential scanning calorimetry. The developed crystals of dipyridamole under optimized conditions ensue in different crystalline habits that significantly improved dissolution rate compared to original dipyridamole. Recrystallized dipyridamole with benzene and acetonitrile, produced needle shaped crystals and the ones recrystallizaed with methanol produced rectangular shaped crystals. Whereas smooth needle shaped crystals were obtained with the methanolic solution of the drug in the presence of Tween-80, Povidone K30 and PEG4000 (AdhiyamanBasu, 2006). Generally, these results were in consistence with the possibility of controlling and enhancing the drug release properties following by a probable improvement in bioavailability of drug particles through characterization of drug crystals. 
Recrystallization of phenytoin in ethanol and acetone by Nokhodchi et al. was shown to produce needle-like and rhombic crystal habits which brought about identical dissolution rates of crystals obtained from both solvents. Considering the results, nature of recrystallization solvents in this case had no effect on dissolution profiles. It was suggested that the differences in dissolution rates for phenytoin was related to the surface area of various crystals with different shapes (Nokhodchi, Bolourtchian et al., 2003). Wettability and the changes in intrinsic dissolution rate of doped phenytoin crystals were evaluated by Chow et al. They stated that the differences in dissolution rates of phenytoin crystalline powders with different morphology were mainly because of the changes in surface area rather than the improvements in the wetting of more polar surface moieties. However, they stated that the areas of the relatively polar faces seem to be valuable determinants of the drug release profiles of doped phenytoin crystals along with the correlation of the surface tensions (Chow et al., 1995).

Talinolol is a cardioselective beta blocker agent that is known to have different crystal structures with strongly differing solubilities when pure water, acetate, or phosphate buffers are employed as dissolution media. Wagner et al. have studied the impact of different dissolution media controlling the crystal structures of talinolol influencing the dissolution rate and solubility of the drug. The crystal structures were analyzed by means of light microscopy, differential scanning calorimetry, and X-ray powder diffraction, detecting the variations of talinolol crystal structures being the source of incomplete and unpredictable nature of the drug bioavailability (Wagner et al., 2003).

Carbamazepine, a routinely used drug in the treatment of epilepsy and trigeminal neuralgia, exists in four polymorphic forms and as a hydrate which could modify the physicochemical properties of the drug. In our previously published paper we established enhanced physicomechanical properties of carbamazepine via recrystallization at different $\mathrm{pH}$ values. The resultant habits of carbamazepine crystals varied from flaky or thin plate-like to needle shape structures which were ascertained using scanning electron microscopy and X-ray powder diffraction. Considering the results of the in vitro dissolution evaluations of carbamazepine samples, a higher dissolution rate for carbamazepine crystals were obtained from media with $\mathrm{pH} 11$ and 1 compared to the original carbamazepine sample. After all, the carbamazepine particles recrystallized from aqueous solutions with different $\mathrm{pH}$ values revealed superior mechanical properties which were generally in consistence with the similar studies of drug recrystallization (Grzesiak et al., 2003; Javadzadeh et al., 2009).

In spite of the absolute potential of drug habit modifications in dissolution rate promotion, far too little attention has been paid to gather detailed documentations of the usage of the approach in enhancement of systemic drug efficiencies following drug bioavailability in human subjects or in suitable animal models. So as to affirm drug recrystallization as an efficient practice in intentionally increasing the bioavailability of poorly soluble drugs, further investigation in this field is mandatory. Ultimately, we could consider the potential management of crystal habits of poorly water soluble drugs as an approach for designing efficient pharmaceutical dosage forms.

\section{Polymorphism}

Very early on, in 1832 Wöhler and Liebig reported the first observation of polymorphism upon cooling a boiling solution of benzamide where needle-shaped crystals would initially 
formed followed by a conversion to rhombic crystals upon standing (WöhlerLiebig, 1832). After a longtime history, polymorphism has maintained its innovation for scientists as a curiosity and an urgent challenge of commercial relevance in manufacturing industries, as well.

In general, polymorphism is known to be the ability of a compound to crystallize in more than one distinct crystal structure. Evidently, structures of different crystals would receive various scopes of the possible ranges of intermolecular interactions like, van der Waals, ionic, and hydrogen bonds. It would not be far from expectations that the different polymorphs of the same molecule will have different free energies affecting all the basically physicochemical properties of the compound, in consequence. Therefore, the crystal structure of drugs have leading signature on both physical and chemical properties in a way that the solid-state forms might demonstrate variations in, color, stability, processability, solubility, dissolution and bioavailability, ranging from the subtle to the severe (RodriguezSpong et al., 2004). Despite the fact that distinct crystal habits have different processing issues or different chemical stability, these variations usually have direct solutions and the real impact of crystal forms is the difference in solubility and bioavailability. Figure 2 gives a fair picture of the probable consequences of a different crystal form in solubility. Decreased solubility of a certain crystalline form of a drug not only brings about lower bioavailability but also reduced rates of drug clearances are inevitable which are the inferences to the safety and efficacy of any drug product.
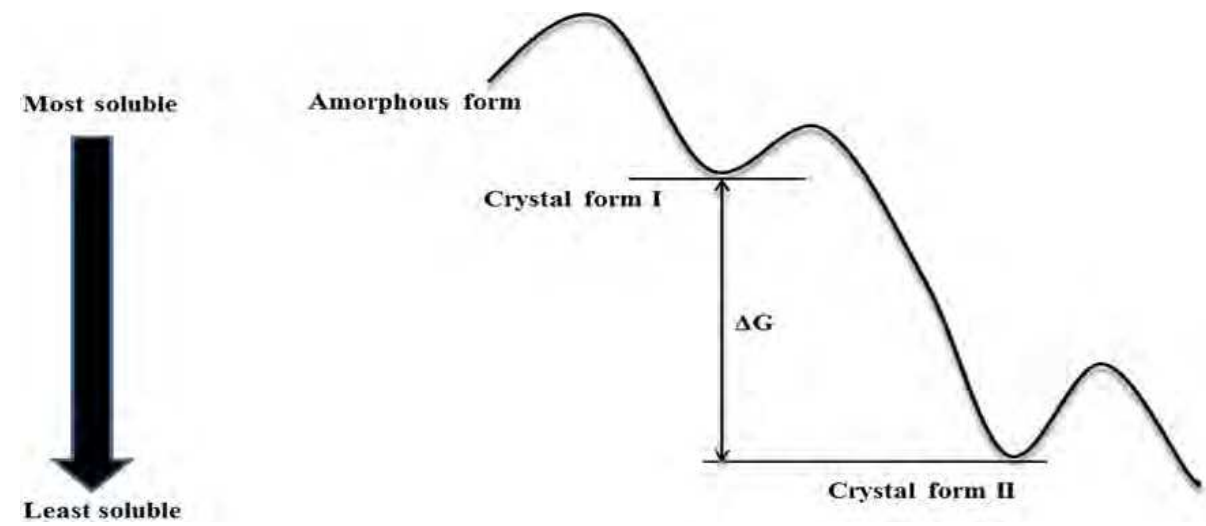

Fig. 2. Relative solubility of different crystal forms as a function of thermodynamic stability

Normally, producing the most thermodynamically stable polymorphic form of a drug in the range of interest is compulsory. The pharmaceutical science has been chiefly in charge for a change in this situation ever since the majority of drugs delivered orally receive rigid approval for a single crystal form or polymorph. Establishing the most thermodynamically stable form of a drug relies on obtaining comprehensive information about the existence and the interrelation of the polymorphs for a given active ingredient. Different polymorphs of a drug could be prepared by recrystallizing the drug with a range of solvents under the optimized conditions which has currently been arisen as an active research province of pharmaceutical science for improving the formulation related problems of drug molecules. 
Accordingly, drug polymorphism investigations have an important role in any reformulation study since it has an impact on the development potential of a drug molecule, so as understanding the polymorphic tendencies of a drug molecule thorough characterization of the observed forms is of prime value.

The first important step in characterizing a polymorph of a drug is to distinguish between different structures of a molecule by its class. As it has been shown in figure 3, the most commonly observed forms in drug solids are the polymorphs, amorphous, crystals, solvates, and hydrates forms, that are fairly tractable from a processing notion. More to the point, hydrates or solvates are considered as pseudo-polymorphs that may either be an entirely different crystalline entity or simply incorporated in the parent crystal lattice; however, it is often possible to remove water or solvate by recrystallization.

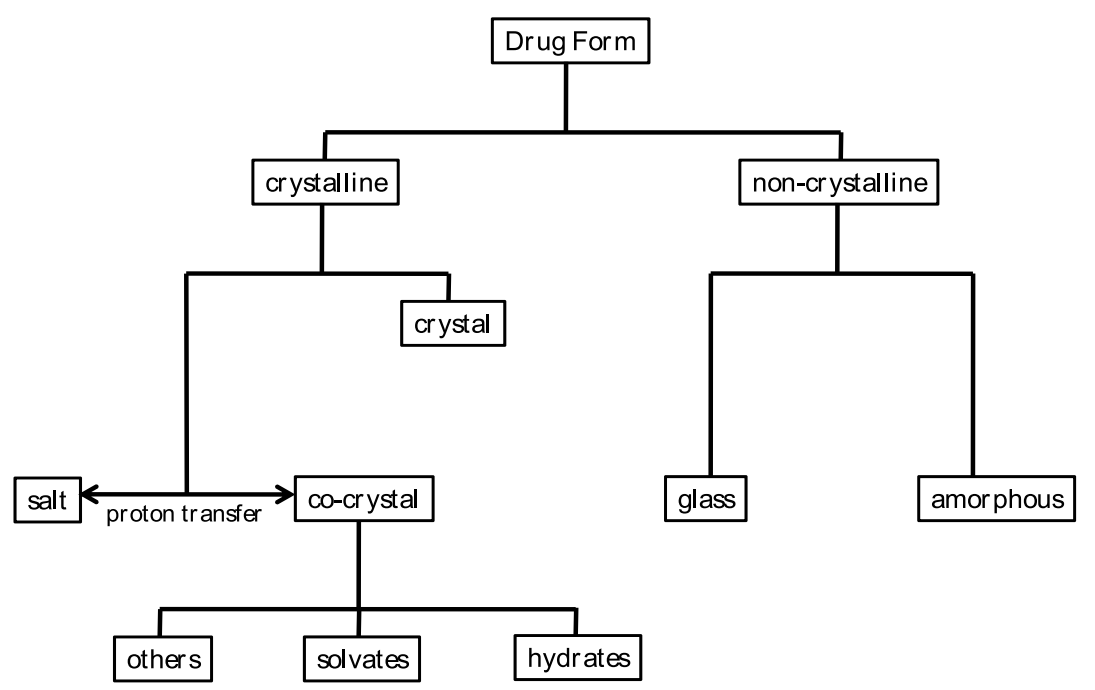

Fig. 3. Pharmaceutical solids in different forms.

While the molecular organization of drugs may differ having impact on the development potential of a drug molecule, the underlying concern for the drug performance is the same. Herein we tried to meet examples and detailed information on how recrystallization was used to improve the relative characteristics of drugs.

Park et al. designed a supercritical anti-solvent process for recrystallization of fluconazole, a triazole antifungal drug which has different polymorphic forms, to modify solid state characteristics of fluconazole by a range of operating conditions such as temperature, pressure, and type of solvent. Fluconazole particles were detected by means of differential scanning calorimetry, thermogravimetry analysis, powder X-ray diffraction, fourier transform infrared spectroscopy and scanning electron microscopy. Regarding the findings of the study, polymorphic forms of fluconazole were remarkably affected by the experimental conditions as the anhydrate form I of drug was obtained at low temperature and the anhydrate form II was obtained at higher temperatures. On the whole they suggested that the solid state characteristics of fluconazole, including the polymorphic form 
could effectively be managed by altering the operating conditions of the recrystallization process such as temperature, pressure, and solvent (Park et al., 2007). In another study, recrystallization of two anti-cancer drugs, erlotinib hydrochloride and fulvestrant was investigated employing the same method of recrystallization by Tien et al. Polymorph conversion from the original form $\mathrm{B}$ to form $\mathrm{E}$ or a prior form $\mathrm{A}$ for erlotinib was demonstrated under appropriate operational conditions which improved the dissolution rate of the drug. The micronized fulvestrant drug particles showed consistent polymorph like the original drug, but with differences in crystal habits. They also confirmed the positive effect of recrystallization in drug modifications (Tien et al., 2010).

Chen et al. evaluated the effects for the type of solvent, temperature and pressure as well as the solution flow rate on sulfathiazole particle formation using the same supercritical antisolvent technology for recrystallization. In the optimum operating condition of acetone as the solvent, temperature at $308 \mathrm{~K}$ with $12 \mathrm{MPa}$ pressure and flow rate of $2 \mathrm{~mL} / \mathrm{min}$ the micronized sulfathiazole including polymorphic forms were obtained. Moreover, it was determined that various solvents resulted in different polymorphisms where the polymorph form III changed to form IV when ethanol was employed as the solvent. As a result, recrystallization of sulfathiazole produced in optimally micronized particles which exhibited a much narrower particle size distribution with an enhanced in vitro dissolution rate by 3.2 times to the original form of the drug (Chen et al., 2010).

\section{Micro-crystal formation}

Apart from the final quality of the drug crystal product in terms of purity, polymorphism, habit and morphology as well as crystal mechanical strength characterized in recrystallization procedure, size distributions of the drug particle is another crucial aspect of the pharmaceutical dosage forms. With reference to the Noyes-Whitney equation, application of a drug substance in a reduced particle size encourages bioavailability of the poorly water-soluble drug substances owing to the enhanced dissolution rate of micron- or nano-size drug particles (Chaumeil, 1998). An alternative area where small sized drug particles are indispensable is the pulmonary drug administration, inhaler drugs, in which drug powders should have a narrow particle size distribution and a mean particle size of 5 $\mu \mathrm{m}$ with almost no particles larger than $10 \mu \mathrm{m}$. Pharmaceutical dosage forms of these kinds are supposed to follow low particle agglomeration tendency, sufficient flow properties, and good batch-to-batch conformity in favor of the relative drug powders (IslamGladki, 2008).

Several techniques could be applied for the preparation of micron sized pharmaceuticals, such as mechanical comminution of the previously formed larger drug particles by crushing, grinding, milling and etc. that are the most common ways in this field. However, these methods not only provide limited opportunity for the control of important drug characteristics, like size, shape, morphology, surface properties but also ascertain distorted drug properties in a principally uncontrolled manner (RasenackMuller, 2004). As the surfaces in mechanically micronized drug powders are not naturally grown as the crystal cleaves at the crystal face and the surface energy changes, processing properties like flowability, agglomeration and stickiness to surfaces are the prevalent phenomenon. Employing milling processes such as jet milling, pearl-ball milling, or high-pressure homogenization other than influencing the preformulation behavior of drug structures, call 
for high energy and manpower being evidence for insufficient coverage of this method for application in reduced particle size drug production. It seems that these commonly being used techniques do not meet the ideal way for the production of small sized drug particles.

Since the suitable physicochemical and biopharmaceutical properties of a drug substance add to the time and cost of drug development, any postern to resolve these problems and produce small particles of drugs in a controlled process maximizes the opportunity to succeed in drug product manufacturing. Unlike the former techniques, production of small particles using controlled production processes such as spray drying, precipitation from supercritical fluid and recrystallization could be applied for the preparation of properly characterized micron sized pharmaceuticals. Microcrystallization in which the solubility and dissolution rate is improved by forming high specific surface area is used for preparation of drug microcrystals by recrystallization methods to reduce the size of the poorly watersoluble drug particles.

The usual technologies for recrystallization are fulfilled in this framework and it is to use solvent change or precipitation method by immediate mixing two liquids in presence of stabilizing agents. Regardless of the absolute efficiency of the recrystallization approach in production of large drug crystals, producing the small drug particles is still a sort of a challenge due to the high surface area of these particles, exerting tendency of a particle growth. So stabilizing agents would be foremost part in this system preventing particle growth by stabilizing the high specific surface area of small particles (LechugaBallesterosRodriguez-Hornedo, 1993). Nevertheless, recrystallization still continues to be one of the important parts of small sized drug production in drug development strategies. Therefore, microcrystals precipitate in presence of stabilizing protective polymers and a large and hydrophilized surface would be formed in a one process step having advantages over traditional milling techniques.

Hence, developing micro-crystallization as an efficient approach that modifies the biopharmaceutical and technological behavior of drug through selection of the process variables to reach an optimal pharmaceutical product has evolved to meet drug development challenges. Exploration on the growing number of publications in domain of micronized drug particle developing techniques apparently to enhance drug dissolution rate, considering the widely increasing number of poorly water-soluble drugs affirms the declaration.

Rasenack et al. prepared microcrystals of a poorly water-soluble drug ECU-01, an antiinflammatory drug in preclinical state of development by a precipitation practice in the presence of stabilizing agents such as gelatin, chitosan, and different types of cellulose ethers and then spray-drying of the formed dispersion. Considering the low specific surface area of the nearly cuboid-like form of ECU-01, the aim of the survey was to enhance the drug dissolution rate by using microcrystals. Precipitation came off through dissolving the drug in acetone followed by an instant pouring an aqueous solution of the stabilizer into the drug solution. Via the use of cellulose type ethers as a stabilizer employed in this technique the thermodynamically unstable small particles were stabilized forming a protective layer on the crystal surface of the homogeneous microcrystals dispersions. Due to the polymorphic nature of the drug, the newly formed crystals appeared in a needle-shaped habit, highly increasing the specific surface area. Consequently, the dissolution rate rose up to $93 \%$ after $20 \mathrm{~min}$ 
compared to the $4 \%$ in common drug, indicating large surface of the microcrystals. Recrystallization of the poorly water-soluble drug ECU-01 was considered as a superior method which is easy to handle and only entails ordinary equipment (Rasenack et al., 2003).

A nonsteroidal anti-inflammatory drug, indomethacin, which has a hydrophobic and $\mathrm{pH}$ dependent solubility nature, was developed and studied by Kim et al. through a microcrystallization technique to improve its physicochemical properties. Microcrystals of indomethacin was produced using a $\mathrm{pH}$-shift procedure in which the drug was dissolved in an alkaline water to prepare saturated indomethacin solution thereafter the $\mathrm{pH}$ of the solution was decreased by adding $0.5 \mathrm{~N}$ hydrochloric acid and stored at $20^{\circ} \mathrm{C}$ for $24 \mathrm{~h}$ to form microcrystal. The findings of the study exhibited similar physicochemical properties for the microcrystals produced and the standard crystalline powder in X-ray diffraction, differential scanning calorimetry, and Fourier transform infrared spectroscopy analyses, exclusive of a lower peak height in X-ray diffraction and somewhat lower melting temperature. The plate-like with uniform sized microcrystals of indomethacin dissolved about twice over the standard crystalline powder in the initial phase of dissolution study. Furthermore, the in vitro biological activity of the indomethacin microcrystals was assessed in their capacity to inhibit the proliferation of colon cancer cells that showed $20 \%$ greater activity than that of the standard crystalline powder. This view might have implications for improving the efficiency of chemotherapy in treating patients with malignant neoplasms using this technique for production of indomethacine microcrystals (Kim et al., 2003).

More recently, Talari et al. evaluated gliclazide microcrystals; a widely used drug for the treatment of non-insulin-dependent diabetes mellitus which shows a low solubility of 55 $\mathrm{mg} / \mathrm{L}$ in water and gastric fluids leading to a low dissolution rate and variable bioavailability. The gliclazide microcrystals were prepared by in situ micronization techniques based on solvent and $\mathrm{pH}$-shift and were examined for the drug absorption and pharmacokinetics of GL after oral administration in rats. Compared to the original drug, scanning electron microscopy showed significant changes in the shape and size of the prepared crystals using both methods. Recrystallized samples not only showed enhanced dissolution rates than untreated drug particles but also a reduced particle size of about 30 and 61 times by solvent-change and $\mathrm{pH}$-shift methods were detected for drug crystals, respectively. Regarding results of the in vivo biological assays for hypoglycemic activity, microcrystallization of gliclazide using both methods resulted in an increased pharmacodynamic effect of glucose-lowering in diabetic rats which could be relevant to the improved dissolution rate of the drug (Talari et al., 2010).

Concisely, the microcrystallization of the drug particulates which has an effect on crystal habit, could also improve the drug absorption characteristics and the subsequent drug bioavailability.

\section{Lyotropic liquid crystals formation}

Liquid crystals (LCs) are a state of matter that has properties between those of a conventional liquid and those of a solid crystal. For instance, an LC may flow like a liquid, but its molecules may be oriented in a crystal-like way. There are many different types of LC phases, which can be distinguished by their different optical properties. When viewed under a microscope using a polarized light source, different liquid crystal phases will 
appear to have distinct textures. The contrasting areas in the textures correspond to domains where the LC molecules are oriented in different directions. Within a domain, however, the molecules are well ordered. LC materials may not always be in an LC phase (just as water may turn into ice or steam).

Liquid crystals can be divided into thermotropic, lyotropic and metallotropic phases. Thermotropic and lyotropic LCs consist of organic molecules. Thermotropic LCs exhibit a phase transition into the LC phase as temperature is changed. Lyotropic LCs exhibit phase transitions as a function of both temperature and concentration of the LC molecules in a solven . Metallotropic LCs are composed of both organic and inorganic molecules; their LC transition depends not only on temperature and concentration, but also on the inorganicorganic composition ratio.

Examples of liquid crystals can be found both in the natural world and in technological applications. Most modern electronic displays are liquid crystal based. Lyotropic liquidcrystalline phases are abundant in living systems. For example, many proteins and cell membranes are LCs. Other well-known LC examples are solutions of soap and various related detergents, as well as the tobacco mosaic virus.

A lyotropic liquid crystal consists of two or more components that exhibit liquid-crystalline properties in certain concentration ranges. In the lyotropic phases, solvent molecules fill the space around the compounds to provide fluidity to the system. In contrast to thermotropic liquid crystals, these lyotropics have another degree of freedom of concentration that enables them to induce a variety of different phases.

A compound, which has two immiscible hydrophilic and hydrophobic parts within the same molecule, is called an amphiphilic molecule. Many amphiphilic molecules show lyotropic liquid-crystalline phase sequences depending on the volume balances between the hydrophilic part and hydrophobic part. These structures are formed through the microphase segregation of two incompatible components on a nanometer scale. Soap is an everyday example of a lyotropic liquid crystal.
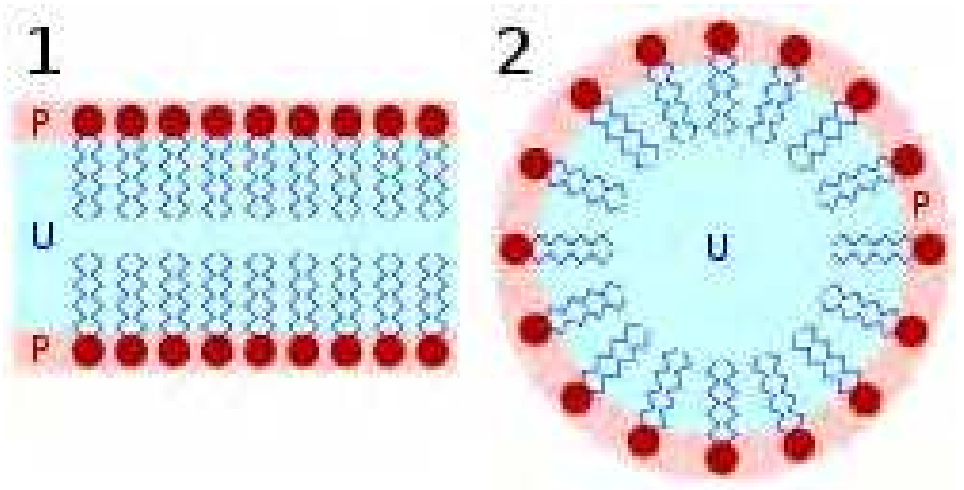

Fig. 4. Structure of lyotropic liquid crystal. The red heads of surfactant molecules are in contact with water, whereas the tails are immersed in oil (blue): bilayer (left) and micelle (right) 
The content of water or other solvent molecules changes the self-assembled structures. At very low amphiphile concentration, the molecules will be dispersed randomly without any ordering. At slightly higher (but still low) concentration, amphiphilic molecules will spontaneously assemble into micelles or vesicles. This is done so as to 'hide' the hydrophobic tail of the amphiphile inside the micelle core, exposing a hydrophilic (water-soluble) surface to aqueous solution. These spherical objects do not order themselves in solution, however. At higher concentration, the assemblies will become ordered. A typical phase is a hexagonal columnar phase, where the amphiphiles form long cylinders (again with a hydrophilic surface) that arrange themselves into a roughly hexagonal lattice. This is called the middle soap phase. At still higher concentration, a lamellar phase (neat soap phase) may form, wherein extended sheets of amphiphiles are separated by thin layers of water. For some systems, a cubic (also called viscous isotropic) phase may exist between the hexagonal and lamellar phases, wherein spheres are formed that create a dense cubic lattice. These spheres may also be connected to one another, forming a bicontinuous cubic phase (VroegeLekkerkerker, 1992).

The objects created by amphiphiles are usually spherical (as in the case of micelles), but may also be disc-like (bicelles), rod-like, or biaxial (all three micelle axes are distinct). These anisotropic self-assembled nano-structures can then order themselves in much the same way as thermotropic liquid crystals do, forming large-scale versions of all the thermotropic phases (such as a nematic phase of rod-shaped micelles).

For some systems, at high concentrations, inverse phases are observed. That is, one may generate an inverse hexagonal columnar phase (columns of water encapsulated by amphiphiles) or an inverse micellar phase (a bulk liquid crystal sample with spherical water cavities).

A generic progression of phases, going from low to high amphiphile concentration, is: Discontinuous cubic phase (micellar cubic phase), Hexagonal phase (hexagonal columnar phase) (middle phase), Lamellar phase, Bicontinuous cubic phase, Reverse hexagonal columnar phase, and Inverse cubic phase (Inverse micellar phase)

Even within the same phases, their self-assembled structures are tunable by the concentration: for example, in lamellar phases, the layer distances increase with the solvent volume. Since lyotropic liquid crystals rely on a subtle balance of intermolecular interactions, it is more difficult to analyze their structures and properties than those of thermotropic liquid crystals. Similar phases and characteristics can be observed in immiscible diblock copolymers.

Lyotropic liquid crystals transitions occur with influence of solvents or recrystallization method. Lyotropic liquid crystals occur as a result of solvent induced aggregation of the constituent mesogens into micellar structure. This types of liquid crystalline states were mostly used for designing the sustain release drug delivery system. It is also used for improving the solubility and stability of insoluble drugs by incorporating it into micellar structure of liquid crystals (Lechuga-Ballesteros et al., 2003).

\section{Spherical crystallization}

Among the particles designed for solid pharmaceutical dosage forms, tablet supports for the half of all oral drug delivery system and $70 \%$ of the all pharmaceutical preparations 
produced. Direct tabletting, simple mixing and compressing drug powders have been widely applied to a large number of drugs on the industrial scale as an excellent technique. A successful tabletting and also compression of any drug material is reliant on the micromeritic properties of the drug crystals. In this context, crystals of needle-shaped or plated-shaped are challengeable to be handled with, due to the poor flowability of these crystals (Kaerger et al., 2004). In 1984 Kawashima et al. introduced crystal agglomeration with controlled properties as spherical crystallization to the pharmaceutical manufacturing and expressed that the spherically dense agglomerates were suitable for direct tabletting. Accordingly, crystallization and agglomeration of the drug substance particles concurrently in one step to transform crystals directly into compacted spherical form during the recrystallization process is defined as spherical crystallization (Kawashima, 1984). Spherical crystals could be established through two different techniques, either by typical spherical crystallization technique or non typical spherical crystallization technique (Nokhodchi et al., 2007). Non typical spherical crystallization technique might be regarded as the traditional crystallization process like salting-out, cooling, precipitation, whereas, the typical spherical crystallization is a threesolvent system employing three solvents; one is the drug dissolution medium known as the good solvent, another is a medium which partially dissolves the drug and has wetting feature that is named bridging liquid, and the last one is immiscible with the drug substance recognized as the bad solvent (Nokhodchi, Maghsoodi et al., 2007; Mahanty et al., 2010).

On average, spherical crystallization techniques are believed to be promising techniques in which the drug crystals are modified using different solvents for direct compressible spherical agglomerates, which can save money and time for tabletting. These approaches not only helps to achieve good flowability, compressibility and micromeritic properties of the drug substances, but also it is known to improve the the wettability, bioavailability, and dissolution rate of some poorly soluble drugs (Kawashima, Handa et al., 1986; Di Martino et al., 1999; NokhodchiMaghsoodi, 2008). Besides, drug materials produced by the spherical crystallization technique result in the economical process in the development of the solid dosage forms for scaling up to a commercial level since it provides reduced time and cost by enabling faster operation, less machinery and fewer personnel.

Seeing that, magnesium aspartate and acetylsalicylic acid crystals in common are tetragonal and prism-shaped with different sizes they show poor flowability and compactibility properties indicating the crystal habit and the electrostatic charge. SzaboRevesz et al. developed magnesium aspartate and acetylsalicylic acid via spherical crystallization since they are used in direct tablet-making and capsule-filling, the particle size and the spherical form are fundamental in view of their processibility. They prepared drug agglomerates through non-typical (magnesium aspartate) and typical (acetylsalicylic acid) spherical crystallization techniques. Crystal agglomerates of these drugs produced by these different spherical crystallization techniques created opportunity for a comparison between the results obtained. Considering the findings of the study, a higher initial cooling rate and a lower stirring rate were favorable in producing crystal agglomerates. The growth of particle size and the spherical form as well as the associated decreased specific surface of the magnesium aspartate and 
acetylsalicylic acid crystal agglomerates created better compactibility and cohesivity characteristics than the control samples in a softer flow time, and a higher bulk density. According to the authors both types of spherical crystallization (non-typical and typical) can be effectively used not only for spherical particle forming but also for size growing of drug materials that are pressed directly into tablets or made into filled capsules without excipients (Szabo-Revesz et al., 2002).

Celecoxib, a non-steroidal anti-inflammatory drug which is the first selective cyclooxygenase-2 inhibitor used in the treatment of osteoarthritis and rheumatoid arthritis exhibits poor flow and compression characteristics as well as incomplete and poor oral bioavailability due to its low aqueous solubility. Variety of papers in this case is representative of the suitability of this drug for spherical crystallization process to enhance the flow, compressibility and solubility properties of seems to be a beneficial objective to improve the following therapeutic efficacy of celecoxib (Banga et al., 2007). Paradkar et al. improved the micromeritic and compressional properties of celecoxib by a spherical crystallization process using the solvent change method. Selection of the solvents depended on the miscibility of solvents and also the solubility of drug in the solvents, which candidated acetone as the good solvent, dichloromethane as the bridging liquid, and water as the bad solvent. A solution of celecoxib in acetone was added to a solution of hydroxy propyl methylcellulose in dichloromethane. Drug was crystallized by adding the solution to wall-baffled vessel containing distilled water followed by a continuous mixing in a controlled speed style creating agglomerated spherical crystals. Celecoxib agglomerates exhibited satisfactory micromeritic, mechanical, and compressional properties demonstrating comparable in vitro drug release performance with the marketed capsule formulation (Paradkar et al., 2002). Elsewhere, Gupta et al. prepared spherical crystals of celecoxib using a more hydrophilic polymer, polyvinylpyrrolidone K-30 (PVP) and acetone, water and chloroform as solvent, nonsolvent and bridging liquid, respectively. The agglomerates were determined by differential scanning calorimetry, X-ray diffraction, IR spectroscopic and scanning electron microscopy. They showed that the crystals possessed a good spherical shape with smooth and regular surface exhibiting significantly improved micromeritic properties compared to pure the drug. Moreover, the aqueous solubility and dissolution rate of the drug from crystals was notably increased nearly two times, with an increase in PVP concentration. In general, this technique may be applicable for producing oral solid dosage forms of other drugs with improved dissolution rate and oral bioavailability (Gupta et al., 2007). Correspondingly, use of these systems has the potential to facilitate drug development by saving valuable time in selecting the optimal physical or chemical characteristics of a given compound.

On the whole, lessening the risk of drug process modifications and providing the opportunity to gain more comprehensive rational property coverage would be established if such information is established through recrystallization processes at an early stage of drug developments. Eventually, it is of the utmost importance to strictly monitor the processing of drug substances with regards to the different crystal habits of drug materials, as well as to obtain a comprehensive understanding of the physical and chemical stability of these polymorphic states. 


\section{References}

Adhiyaman, R. \& Basu, S.K. (2006). Crystal modification of dipyridamole using different solvents and crystallization conditions. International Journal of Pharmaceutics, Vol.321, No.1-2, pp. 27-34, ISSN 03785173

Adibkia, K., Hamedeyazdan, S. \& Javadzadeh, Y. (2011). Drug release kinetics and physicochemical characteristics of floating drug delivery systems. Expert Opin Drug Deliv, Vol.8, No.7, pp. 891-903, ISSN 1744-7593

Banga, S., Chawla, G., Varandani, D., Mehta, B.R. \& Bansal, A.K. (2007). Modification of the crystal habit of celecoxib for improved processability. J Pharm Pharmacol, Vol.59, No.1, pp. 29-39, ISSN 0022-3573

Blagden, N., de Matas, M., Gavan, P.T. \& York, P. (2007). Crystal engineering of active pharmaceutical ingredients to improve solubility and dissolution rates. Adv Drug Deliv Rev, Vol.59, No.7, pp. 617-630, ISSN 0169-409X

Carino, S.R., Sperry, D.C. \& Hawley, M. (2006). Relative bioavailability estimation of carbamazepine crystal forms using an artificial stomach-duodenum model. J Pharm Sci, Vol.95, No.1, pp. 116-125, ISSN 0022-3549

Chaumeil, J.C. (1998). Micronization: a method of improving the bioavailability of poorly soluble drugs. Methods Find Exp Clin Pharmacol, Vol.20, No.3, pp. 211-215, ISSN 0379-0355

Chen, J. \& Trout, B.L. (2008). Computational study of solvent effects on the molecular self-assembly of tetrolic acid in solution and implications for the polymorph formed from crystallization. J Phys Chem B, Vol.112, No.26, pp. 7794-7802, ISSN 1520-6106

Chen, Y.-M., Tang, M. \& Chen, Y.-P. (2010). Recrystallization and micronization of sulfathiazole by applying the supercritical antisolvent technology. Chemical Engineering Journal, Vol.165, No.1, pp. 358-364, ISSN 13858947

Chow, A.H.L., Hsia, C.K., Gordon, J.D., Young, J.W.M. \& Vargha-Butleff, E.I. (1995). Assessment of wettability and its relationship to the intrinsic dissolution rate of doped phenytoin crystals. International Journal of Pharmaceutics, Vol.126 pp. 21-28, ISSN 0378-5173

Dahlberg, C., Dvinskikh, S.V., Schuleit, M. \& Furo, I. (2011). Polymer Swelling, Drug Mobilization and Drug Recrystallization in Hydrating Solid Dispersion Tablets Studied by Multinuclear NMR Microimaging and Spectroscopy. Mol Pharm, Vol.8, No.4, pp. 1247-1256, ISSN 1543-8392

Di Martino, P., Barthelemy, C., Piva, F., Joiris, E., Palmieri, G.F. \& Martelli, S. (1999). Improved dissolution behavior of fenbufen by spherical crystallization. Drug Dev Ind Pharm, Vol.25, No.10, pp. 1073-1081, ISSN 0363-9045

Grzesiak, A.L., Lang, M., Kim, K. \& Matzger, A.J. (2003). Comparison of the four anhydrous polymorphs of carbamazepine and the crystal structure of form I. J Pharm Sci, Vol.92, No.11, pp. 2260-2271, ISSN 0022-3549

Gupta, V.R., Mutalik, S., Patel, M.M. \& Jani, G.K. (2007). Spherical crystals of celecoxib to improve solubility, dissolution rate and micromeritic properties. Acta Pharm, Vol.57, No.2, pp. 173-184, ISSN 1330-0075 
Harbury, L. (1947). Solubility and melting point as functions of particle size; the induction period of crystallization. J Phys Colloid Chem, Vol.51, No.2, pp. 382-391, ISSN 00927023

Heng, J.Y., Bismarck, A. \& Williams, D.R. (2006). Anisotropic surface chemistry of crystalline pharmaceutical solids. AAPS PharmSciTech, Vol.7, No.4, pp. 84, ISSN 1530-9932

Islam, N. \& Gladki, E. (2008). Dry powder inhalers (DPIs)--a review of device reliability and innovation. Int J Pharm, Vol.360, No.1-2, pp. 1-11, ISSN 0378-5173

Jamali, F. \& Mitchell, A.G. (1973). The recrystallization and dissolution of acetylsalicylic acid. Acta Pharm Suec, Vol.10, No.4, pp. 343-352, ISSN 0001-6675

Javadzadeh, Y., Mohammadi, A., Khoei, N.S. \& Nokhodchi, A. (2009). Improvement of physicomechanical properties of carbamazepine by recrystallization at different $\mathrm{pH}$ values. Acta Pharm, Vol.59, No.2, pp. 187-197, ISSN 1330-0075

Jozwiakowski, M.J., Nguyen, N.A., Sisco, J.M. \& Spancake, C.W. (1996). Solubility behavior of lamivudine crystal forms in recrystallization solvents. J Pharm Sci, Vol.85, No.2, pp. 193-199, ISSN 0022-3549

Kaerger, J.S., Edge, S. \& Price, R. (2004). Influence of particle size and shape on flowability and compactibility of binary mixtures of paracetamol and microcrystalline cellulose. Eur J Pharm Sci, Vol.22, No.2-3, pp. 173-179, ISSN 0928-0987

Kawashima, Y. (1984). Development of spherical crystallization technique and its application to pharmaceutical systems. Arch Pharm. Res., Vol.7, No.2, pp. 145151

Kawashima, Y. (2003). Improved flowability and compactibility of spherically agglomerated crystals of ascorbic acid for direct tableting designed by spherical crystallization process. Powder Technology, Vol.130, No.1-3, pp. 283-289, ISSN 00325910

Kawashima, Y., Handa, T., Takeuchi, H., Okumura, M., Katou, H. \& Nagata, O. (1986). Crystal modification of phenytoin with polyethylene glycol for improving mechanical strength, dissolution rate and bioavailability by a spherical crystallization technique. Chem Pharm Bull (Tokyo), Vol.34, No.8, pp. 3376-3383, ISSN 0009-2363

Kim, S.T., Kwon, J.H., Lee, J.J. \& Kim, C.W. (2003). Microcrystallization of indomethacin using a pH-shift method. Int J Pharm, Vol.263, No.1-2, pp. 141-150, ISSN 03785173

Kobayashi, Y., Ito, S., Itai, S. \& Yamamoto, K. (2000). Physicochemical properties and bioavailability of carbamazepine polymorphs and dihydrate. Int J Pharm, Vol.193, No.2, pp. 137-146, ISSN 0378-5173

Krishnaiah S.R., Y. (2010). Pharmaceutical Technologies for Enhancing Oral Bioavailability of Poorly Soluble Drugs. Journal of Bioequivalence E Bioavailability, Vol.02, No.02, pp. 28-36, ISSN 0975-0851

Krishnaiah, Y.S.R. (2010). Pharmaceutical technologies for enhancing oral bioavailability of poorly soluble drugs. Journal of Bioequivalence \& Bioavailability, Vol.2, No.2, pp. 028036, ISSN 0975-0851

Lechuga-Ballesteros, D., Abdul-Fattah, A., Stevenson, C.L. \& Bennett, D.B. (2003). Properties and stability of a liquid crystal form of cyclosporine-the first reported naturally 
occurring peptide that exists as a thermotropic liquid crystal. J Pharm Sci, Vol.92, No.9, pp. 1821-1831, ISSN 0022-3549

Lechuga-Ballesteros, D. \& Rodriguez-Hornedo, N. (1993). Growth and morphology of Lalanine crystals: influence of additive adsorption. Pharm Res, Vol.10, No.7, pp. 10081014, ISSN 0724-8741

Liebenberg, W., de Villiers, M.M., Wurster, D.E., Swanepoel, E., Dekker, T.G. \& Lotter, A.P. (1999). The effect of polymorphism on powder compaction and dissolution properties of chemically equivalent oxytetracycline hydrochloride powders. Drug Dev Ind Pharm, Vol.25, No.9, pp. 1027-1033, ISSN 0363-9045

Llinas, A., Burley, J.C., Box, K.J., Glen, R.C. \& Goodman, J.M. (2007). Diclofenac solubility: independent determination of the intrinsic solubility of three crystal forms. J Med Chem, Vol.50, No.5, pp. 979-983, ISSN 0022-2623

Lobenberg, R. \& Amidon, G.L. (2000). Modern bioavailability, bioequivalence and biopharmaceutics classification system. New scientific approaches to international regulatory standards. Eur J Pharm Biopharm, Vol.50, No.1, pp. 3-12, ISSN 0939-6411

Maghsoodi, M., Hassan-Zadeh, D., Barzegar-Jalali, M., Nokhodchi, A. \& Martin, G. (2007). Improved compaction and packing properties of naproxen agglomerated crystals obtained by spherical crystallization technique. Drug Dev Ind Pharm, Vol.33, No.11, pp. 1216-1224, ISSN 0363-9045

Mahanty, S., Sruti, J., Niranjan Patra, C. \& Bhanoji Rao, M.E. (2010). Particle design of drugs by spherical crystallization techniques. International Journal of Pharmaceutical Sciences and Nanotechnology, Vol.3, No.2, pp. 912-918, ISSN 0974 9446

Mirmehrabi, M. \& Rohani, S. (2005). An approach to solvent screening for crystallization of polymorphic pharmaceuticals and fine chemicals. J Pharm Sci, Vol.94, No.7, pp. 1560-1576, ISSN 0022-3549

Nokhodchi, A., Bolourtchian, N. \& Dinarvand, R. (2003). Crystal modification of phenytoin using different solvents and crystallization conditions. Int J Pharm, Vol.250, No.1, pp. 85-97, ISSN 0378-5173

Nokhodchi, A. \& Maghsoodi, M. (2008). Preparation of spherical crystal agglomerates of naproxen containing disintegrant for direct tablet making by spherical crystallization technique. AAPS PharmSciTech, Vol.9, No.1, pp. 54-59, ISSN 15309932

Nokhodchi, A., Maghsoodi, M. \& Hassanzadeh, D. (2007). An Improvement of Physicomechanical Properties of Carbamazepine Crystals. Iranian Journal of Pharmaceutical Research, Vol.6, No.2, pp. 83-89, ISSN 1735-0328

Paradkar, A.R., Pawar, A.P., Chordiya, J.K., Patil, V.B. \& Ketkar, A.R. (2002). Spherical crystallization of celecoxib. Drug Dev Ind Pharm, Vol.28, No.10, pp. 1213-1220, ISSN 0363-9045

Park, H.J., Kim, M.S., Lee, S., Kim, J.S., Woo, J.S., Park, J.S. \& Hwang, S.J. (2007). Recrystallization of fluconazole using the supercritical antisolvent (SAS) process. Int J Pharm, Vol.328, No.2, pp. 152-160, ISSN 0378-5173

Perlovich, G.L., Strakhova, N.N., Kazachenko, V.P., Volkova, T.V., Tkachev, V.V., Schaper, K.J. \& Raevsky, O.A. (2008). Sulfonamides as a subject to study 
molecular interactions in crystals and solutions: sublimation, solubility, solvation, distribution and crystal structure. Int J Pharm, Vol.349, No.1-2, pp. 300313, ISSN 0378-5173

Rasenack, N., Hartenhauer, H. \& Muller, B.W. (2003). Microcrystals for dissolution rate enhancement of poorly water-soluble drugs. Int J Pharm, Vol.254, No.2, pp. 137-145, ISSN 0378-5173

Rasenack, N. \& Muller, B.W. (2004). Micron-size drug particles: common and novel micronization techniques. Pharm Dev Technol, Vol.9, No.1, pp. 1-13, ISSN 10837450

Rodriguez-Spong, B., Price, C.P., Jayasankar, A., Matzger, A.J. \& Rodriguez-Hornedo, N. (2004). General principles of pharmaceutical solid polymorphism: a supramolecular perspective. Adv Drug Deliv Rev, Vol.56, No.3, pp. 241-274, ISSN 0169-409X

Seton, L., Roberts, M. \& Ur-Rehman, F. (2010). Compaction of recrystallised ibuprofen. Chemical Engineering Journal, Vol.164, No.2-3, pp. 449-452, ISSN 1385-8947

Sinclair, W., Leane, M., Clarke, G., Dennis, A., Tobyn, M. \& Timmins, P. (2011). Physical stability and recrystallization kinetics of amorphous ibipinabant drug product by fourier transform raman spectroscopy. J Pharm Sci, pp., ISSN 1520-6017

Suzuki, T., Araki, T., Kitaoka, H. \& Terada, K. (2010). Studies on mechanism of thermal crystal transformation of sitafloxacin hydrates through melting and recrystallization, yielding different anhydrates depending on initial crystalline forms. Int J Pharm, Vol.402, No.1-2, pp. 110-116, ISSN 1873-3476

Szabo-Revesz, P., Hasznos-Nezdei, M., Farkas, B., Goczo, H., Pintye-Hodi, K. \& Eros, I. (2002). Crystal growth of drug materials by spherical crystallization. Journal of Crystal Growth, Vol.237, No.239, pp. 2240-2245, ISSN 0022-0248

Talari, R., Varshosaz, J., Mostafavi, S.A. \& Nokhodchi, A. (2010). Gliclazide microcrystals prepared by two methods of in situ micronization: pharmacokinetic studies in diabetic and normal rats. AAPS PharmSciTech, Vol.11, No.2, pp. 786-792, ISSN 15309932

Tian, F., Sandler, N., Aaltonen, J., Lang, C., Saville, D.J., Gordon, K.C., Strachan, C.J., Rantanen, J. \& Rades, T. (2007). Influence of polymorphic form, morphology, and excipient interactions on the dissolution of carbamazepine compacts. J Pharm Sci, Vol.96, No.3, pp. 584-594, ISSN 0022-3549

Tien, Y.-C., Su, C.-S., Lien, L.-H. \& Chen, Y.-P. (2010). Recrystallization of erlotinib hydrochloride and fulvestrant using supercritical antisolvent process. The Journal of Supercritical Fluids, Vol.55, No.1, pp. 292-299, ISSN 0896-8446

Tiwary, A.K. (2001). Modification of crystal habit and its role in dosage form performance. Drug Dev Ind Pharm, Vol.27, No.7, pp. 699-709, ISSN 0363-9045

Vroege, G.J. \& Lekkerkerker, H.N.W. (1992). Phase transitions in lyotropic colloidal and polymer liquid crystals. Rep. Fmg. Phys., Vol.55, pp. 1241-1309, ISSN 00344885

Wagner, D., Glube, N., Berntsen, N., Tremel, W. \& Langguth, P. (2003). Different dissolution media lead to different crystal structures of talinolol with impact on its dissolution and solubility. Drug Dev Ind Pharm, Vol.29, No.8, pp. 891-902, ISSN 0363-9045 
Wöhler, F. \& Liebig, J. (1832). Untersuchungen über das Radikal der Benzoesäure. Annalen der Pharmacie, Vol.3, No.3, pp. 249-282, ISSN 0075-4617

Yeo, S., Kim, M. \& Lee, J. (2003). Recrystallization of sulfathiazole and chlorpropamide using the supercritical fluid antisolvent process. The Journal of Supercritical Fluids, Vol.25, No.2, pp. 143-154, ISSN 0896-8446 


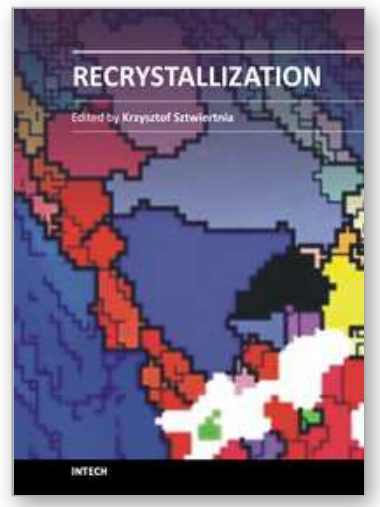

\author{
Recrystallization \\ Edited by Prof. Krzysztof Sztwiertnia
}

ISBN 978-953-51-0122-2

Hard cover, 464 pages

Publisher InTech

Published online 07, March, 2012

Published in print edition March, 2012

Recrystallization shows selected results obtained during the last few years by scientists who work on recrystallization-related issues. These scientists offer their knowledge from the perspective of a range of scientific disciplines, such as geology and metallurgy. The authors emphasize that the progress in this particular field of science is possible today thanks to the coordinated action of many research groups that work in materials science, chemistry, physics, geology, and other sciences. Thus, it is possible to perform a comprehensive analysis of the scientific problem. The analysis starts from the selection of appropriate techniques and methods of characterization. It is then combined with the development of new tools in diagnostics, and it ends with modeling of phenomena.

\title{
How to reference
}

In order to correctly reference this scholarly work, feel free to copy and paste the following:

Yousef Javadzadeh, Sanaz Hamedeyazdan and Solmaz Asnaashari (2012). Recrystallization of Drugs: Significance on Pharmaceutical Processing, Recrystallization, Prof. Krzysztof Sztwiertnia (Ed.), ISBN: 978-95351-0122-2, InTech, Available from: http://www.intechopen.com/books/recrystallization/recrystallization-ofdrugs-significance-on-pharmaceutical-processing

\section{INTECH}

open science | open minds

\section{InTech Europe}

University Campus STeP Ri Slavka Krautzeka 83/A 51000 Rijeka, Croatia Phone: +385 (51) 770447 Fax: +385 (51) 686166 www.intechopen.com

\author{
InTech China \\ Unit 405, Office Block, Hotel Equatorial Shanghai \\ No.65, Yan An Road (West), Shanghai, 200040, China \\ 中国上海市延安西路65号上海国际贵都大饭店办公楼405单元 \\ Phone: +86-21-62489820 \\ Fax: +86-21-62489821
}


(C) 2012 The Author(s). Licensee IntechOpen. This is an open access article distributed under the terms of the Creative Commons Attribution 3.0 License, which permits unrestricted use, distribution, and reproduction in any medium, provided the original work is properly cited. 